\title{
Diameter and girth of Torsion Graph
}

\author{
P. Malakooti Rad 1, S. Yassemi 2, Sh. Ghalandarzadeh 3, P. Safari
}

\begin{abstract}
Let $R$ be a commutative ring with identity. Let $M$ be an $R$-module and $T(M)^{*}$ be the set of nonzero torsion elements. The set $T(M)^{*}$ makes up the vertices of the corresponding torsion graph, $\Gamma_{R}(M)$, with two distinct vertices $x, y \in T(M)^{*}$ forming an edge if $\operatorname{Ann}(x) \cap A n n(y) \neq 0$. In this paper we study the case where the graph $\Gamma_{R}(M)$ is connected with $\operatorname{diam}\left(\Gamma_{R}(M)\right) \leq 3$ and we investigate the relationship between the diameters of $\Gamma_{R}(M)$ and $\Gamma_{R}(R)$. Also we study girth of $\Gamma_{R}(M)$, it is shown that if $\Gamma_{R}(M)$ contains a cycle, then $\operatorname{gr}\left(\Gamma_{R}(M)\right)=3$.
\end{abstract}

\section{INTRODUCTION}

Let $R$ be a commutative ring with identity and $M$ a unitary $R$-module. The idea of associating a graph with the zero-divisors of a commutative ring was introduced by Beck in [10], where the author talked about the colorings of such graphs. He lets every elements of $R$ is a vertex in the graph, and two vertices $x, y$ are adjacent if and only if $x y=0$. In [5], Anderson and Livingston introduced and studied the zero-divisor graph whose vertices are non-zero zero-divisors while $x-y$ is an edge whenever $x y=0$. Anderson and Badawi also introduced and investigated total graph of commutative ring in $[1,2]$. The zero-divisor graph of a commutative ring has been studied extensively by several authors $[3,4,6,9,14,15,16]$. The concept of zero-divisor graph has been extended to non-commutative rings by Redmond [17].

Key Words: Diameter, Girth, Multiplication modules, Torsion graphs.

2010 Mathematics Subject Classification: 13A99; 05C99;13C99

Received:12 June, 2013

Revised: 30 August, 2013

Accepted: 24 September, 2013 
Let $x \in M$. The residual of $R x$ by $M$ denoted by $[x: M]=\{r \in R \mid r M \subseteq$ $R x\}$. The annihilator of an $R$-module $M$, denoted by $A n n_{R}(M)=[0: M]$. If $m \in M$, then $\operatorname{Ann}(m)=\{r \in R \mid r m=0\}$. Let $T(M)=\{m \in M \mid \operatorname{Ann}(m)=$ $0\}$. It is clear that if $R$ is an integral domain then $T(M)$ is a submodule of $M$, which is called torsion submodule of $M$. If $T(M)=0$ then the module $M$ is said torsion-free, and it is called a torsion module if $T(M)=M$.

In this paper, we investigate the concept of torsion-graph for modules as a natural generalization of zero-divisor graph for rings. Here the torsion graph $\Gamma_{R}(M)$ of $M$ is a simple graph whose vertices are non-zero torsion elements of $M$ and two different elements $x, y$ are adjacent if and only if $\operatorname{Ann}(x) \cap A n n(y) \neq$ 0 . Thus $\Gamma_{R}(M)$ is an empty graph if and only if $M$ is a torsion-free $R$-module. Clearly if $R$ is a domain or $A n n(M) \neq 0$, then $\Gamma_{R}(M)$ is complete. This study helps to illuminate the structure of $T(M)$, for example, if $M$ is a multiplication $R$-module, we show that $M$ is finite if and only if $\Gamma_{R}(M)$ is finite.

Recall that a simple graph is finite if its vertices set is finite, and we use the symbol $\left|\Gamma_{R}(M)\right|$ to denote the number of vertices in graph $\Gamma_{R}(M)$. Also, a graph $G$ is connected if there is a path between any two distinct vertices. The distance, $d(x, y)$ between connected vertices $x, y$ is the length of the shortest path from $x$ to $y,(d(x, y)=\infty$ if there is no such path). An isolated vertex is a vertex that has no edges incident to it. The diameter of $G$ is the diameter of connected graph which is the supremum of the distance between vertices. The diameter is zero if the graph consist of a single vertex. The girth of $G$, denoted by $\operatorname{gr}(G)$ is defined as the length of the shortest cycle in $G,(\operatorname{gr}(G)=\infty$ if $G$ contains no cycle). A complete graph is a simple graph whose vertices are pairwise adjacent, the complete graph with $n$ vertices is denoted $K_{n}$.

A ring $R$ is called reduced if $\operatorname{Nil}(R)=0$. A ring $R$ is von Neumann regular if for each $a \in R$, there exists an element $b \in R$ such that $a=a^{2} b$. It is clear that every von Neumann regular ring is reduced.

One may address three major problem in this area: characterization of the resulting graphs, characterization of module with isomorphic graphs, and realization of the connection between the structures of a module and the corresponding graph, in this paper we focus on the third problem.

The organization of this paper is as follows:

In section 2 , we study the torsion graph of finite multiplication module, we show that if the torsion graph of multiplication $R$-module $M$ is finite(when $\Gamma_{R}(M)$ is not empty ) then $M$ is finite, specially if $\Gamma_{R}(M)$ has an isolated vertex, then $M \cong M_{1} \oplus M_{2}$, in which $M_{1}, M_{2}$ are simple submodule of $M$.

In section 3 , we show that $\Gamma_{R}(M)$ is connected with $\operatorname{diam}\left(\Gamma_{R}(M)\right) \leq 3$ if one of the following hold:

(1) $\Gamma_{R}(R)$ is a complete graph.

(2) $R$ be a von Neumann regular ring and $R \neq A n n(x) \oplus A n n(y)$ for any 
$x, y \in T(M)^{*}$.

(3) $\operatorname{Nil}((R) \neq 0$.

In section 4 , we study the girth of torsion graph for an $R$-module $M$. It is shown that if $\Gamma_{R}(M)$ contains a cycle, then $\operatorname{gr}\left(\Gamma_{R}(M)\right)=3$

We follow standard notation and terminology from graph theory [12] and module theory [8].

\section{Properties of torsion graph}

This section is concerned with some basic and important results in the theory of torsion graphs over a module.

The following examples show that non-isomorphic modules may have the same torsion graph.

Example 2.1. Let $M=M_{1} \oplus M_{2}$ be an $R$-module, where $M_{1}$ is a torsion-free module. So $T(M)^{*}=\left\{\left(0, m_{2}\right) \mid m_{2} \in T\left(M_{2}\right)^{*}\right\}$. Below are the torsion graphs for some $\mathbb{Z}$-modules and ring $R$ as $R$-modules.

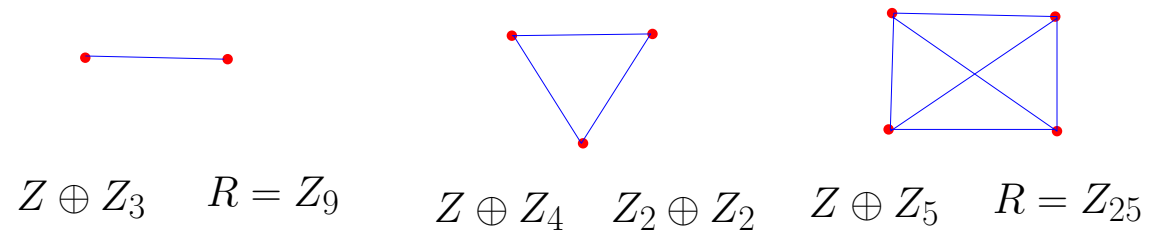

Lemma 2.2. If $R$ is an integral domain, then $\Gamma_{R}(M)$ is complete.

Proof. Let $R$ be an integral domain and $x, y \in T(M)^{*}$, so there is non-zero element $r, s \in R$ such that $r x=s y=0$. Since $R$ is an integral domain, $0 \neq r s \in A n n(x) \cap \operatorname{Ann}(y)$. Thus $d(x, y)=1$ and $\Gamma_{R}(M)$ is complete.

Before we go on discussing the other properties of $\Gamma_{R}(M)$, we give, the following theorem shows that for a multiplication $R$ - module $M, \Gamma_{R}(M)$ is finite (except, when $\Gamma_{R}(M)$ is empty) if and only if $M$ is finite.

Theorem 2.3. Let $M$ be an R-module with Ann $(x)=A n n([x: M] M)$ for all $x \in T(M)^{*}$. Then $\Gamma_{R}(M)$ is finite if and only if either $M$ is finite or $M$ is a torsion free $R$-module.

Proof. Suppose that $\Gamma_{R}(M)$ is finite and nonempty. Let $x \in T(M)^{*}$, hence there is $0 \neq s \in[x: M]$. Let $N=[x: M] M$, so $0 \neq A n n(x) \subseteq \operatorname{Ann}(n)$ for all $n \in N$, thus $N \subseteq T(M)^{*}$, therefore $N$ is finite. Now if $M$ is infinite, then there is a $n \in N$ with $H=\{m \in M \mid s m=n\}$ infinite, then for all distinct 
elements $m_{1}, m_{2} \in H, s \in A n n\left(m_{1}-m_{2}\right)$. So $m_{1}-m_{2} \in T(M)^{*}$, which is a contradiction, therefore $M$ be finite.

In the following example, it is shown that the condition $\operatorname{Ann}(x)=\operatorname{Ann}([x$ : $M] M$ ) for all $x \in T(M)^{*}$ in the above Theorem cannot be omitted.

Example 2.4. Let $R=\mathbb{Z}$ and $M=\mathbb{Z} \oplus \mathbb{Z}_{3}$. Clearly $M$ is not finite, but $V\left(\Gamma_{R}(M)\right)=\{(0, \overline{1}),(0, \overline{2})\}$ and so $\Gamma_{R}(M)$ is finite.

Corollary 2.5. Let $M$ be a multiplication $R$-module. Then $\Gamma_{R}(M)$ is finite if and only if either $M$ is finite or $M$ is a torsion free $R$-module.

Theorem 2.6. Let $M$ be a multiplication R-module. If $\Gamma_{R}(M)$ has an isolated vertex, then $M=M_{1} \oplus M_{2}$ is a faithful $R$-module, where $M_{1}$ and $M_{2}$ are two submodules of $M$ such that $M_{1}$ has only two elements. Especially, if $M$ is finite then $M_{2}$ is simple.

Proof. Suppose that $x \in T(M)^{*}$ be an isolated vertex, so for all $y \in T(M)^{*}$ we have $\operatorname{Ann}(x) \cap A n n(y)=0$ and $M$ is faithful. If $R x \cap R y=0$, then there is vertex $z \in R x \cap R y$ that is adjacent to $x$, which is a contradiction. Thus $[x: M] y \in R x \cap R y=0$. If $[x: M] x=0$, then $[x: M] \in \operatorname{Ann}(x) \cap \operatorname{Ann}(y)$, which is a contradiction. Therefore $[x: M] x \neq 0$ and there is $\alpha \in[x: M]$ such that $\alpha x \neq 0$. Since $x$ is an isolated vertex $R x=\{0, x\}$, thus $\alpha x=x$. One can easily check that $M=R x+A n n(x) M$. Now suppose that $w \in R x \cap A n n(x) M$, thus $w=r x$ for some $r \in R$, hence $\alpha w=r \alpha x=r x=w$ and so $w=r \alpha x \in$ $\operatorname{Ann}(x) \alpha M=0$. Therefore $M=M_{1} \oplus M_{2}$, in which $\left|M_{1}\right|=|R x|=2$.

Now, suppose that $M$ be a finite multiplication $R$-module. Since $M=$ $M_{1} \oplus M_{2}$, we have $M_{2}$ is finite and so $M_{2}$ is an Artinian $R$-module, Also by Theorem 2.2 and Corollary 2.9 [13], $M_{2}$ is cyclic, so $M_{2} \cong \frac{R}{A n n\left(M_{2}\right)}$. Assume that

$$
D\left(M_{2}\right)=\left\{m_{2} \in M_{2} \mid\left[m_{2}: M\right]\left[m_{2}^{\prime}: M\right] M=0\right\} .
$$

We claim that $D\left(M_{2}\right)=0$. If $D\left(M_{2}\right) \neq 0$, then there is a $0 \neq m_{2} \in M_{2}$, such that

$$
\left[m_{2}: M\right]\left[m_{2}^{\prime}: M\right] M=0
$$

for some $0 \neq m_{2}^{\prime} \in M_{2}$. Thus $\alpha m_{2}=0$ for some non-zero element $\alpha \in\left[m_{2}^{\prime}\right.$ : $M]$. Also $\alpha x \in R x \cap M_{2}=0$, so $\alpha\left(m_{2}+x\right)=0=\alpha x$, which is a contradiction, consequently $D\left(M_{2}\right)=0$. Now we show that $\operatorname{Ann}\left(M_{2}\right)$ is prime ideal of $R$. Let $s t \in \operatorname{Ann}\left(M_{2}\right)$ for $s, t \in R$. So $s t M_{2}=0$, hence

$$
\left[s M_{2}: M\right]\left[t M_{2}: M\right] M=0 .
$$

Since $D\left(M_{2}\right)=0$, we have $s M_{2}=t M_{2}=0$. thus $\operatorname{Ann}\left(M_{2}\right)$ is prime ideal of $R$. Hence $\frac{R}{\operatorname{Ann}\left(M_{2}\right)}$ is a finite integral domain and so is a field, thus $\operatorname{Ann}\left(M_{2}\right)$ is a maximal ideal of $R$. Therefore $M_{2}$ is a simple $R$-module. 


\section{$3 \quad$ Diameter of torsion graph}

In this section, we investigate the relationship between the diameter of $\Gamma_{R}(M)$ and $\Gamma_{R}(R)$. First, we study the case where $\Gamma_{R}(M)$ is connected with diameter $\leq 3$.

Theorem 3.1. Let $M$ be an $R$-module. Then $\Gamma_{R}(M)$ is connected with $\operatorname{diam}\left(\Gamma_{R}(M)\right) \leq 3$ if one of the following hold:

(1) $\Gamma_{R}(R)$ is a complete graph.

(2) $R$ be a von Neumann regular ring and $R \neq A n n(x) \oplus A n n(y)$ for any $x, y \in T(M)^{*}$.

(3) $\operatorname{Nil}((R) \neq 0$.

Proof. $\quad$ Let $x, y \in T(M)^{*}$ be two distinct elements. If $\operatorname{Ann}(x) \cap A n n(y) \neq$ 0 or $A n n(M) \neq 0$, then $d(x, y)=1$. Therefore we suppose that $M$ is faithful and $\operatorname{Ann}(x) \cap A n n(y)=0$. So there are two non-zero elements $s, t \in R$ such that $s x=t y=0$ but $s y \neq 0, t x \neq 0$.

(1) Suppose that $\Gamma_{R}(R)$ is a complete graph, hence $\operatorname{Ann}(s) \cap \operatorname{Ann}(t) \neq$ 0 , so $x-t x-s y-y$ is a path of length 3 . Hence $d(x, y) \leq 3$, thus $\operatorname{diam}\left(\Gamma_{R}(M)\right) \leq 3$.

(2) Let $R$ is a von Neumann regular ring. We know $s=u_{1} e_{1}$ and $t=u_{2} e_{2}$ for some non-zero idempotent elements $e_{1}, e_{2}$ and unit elements $u_{1}, u_{2}$ such that $\left(1-e_{1}\right)\left(1-e_{2}\right) \in A n n(s) \cap A n n(t)$. If $A n n(s) \cap A n n(t)=0$, then $1 \in R s+R t \subseteq A n n(x) \cap A n n(y)$, hence $R \cong A n n(x) \oplus A n n(y)$, which is a contradiction. Therefore $A n n(s) \cap A n n(t) \neq 0$ and $x-t x-s y-y$ is a path of length 3 , so $d(x, y) \leq 3$. Thus $\operatorname{diam}\left(\Gamma_{R}(M)\right) \leq 3$.

(3) Let $0 \neq a \in \operatorname{Nil}(R)$, so $a^{n}=0$ and $a^{n-1} \neq 0$ for some $n \in \mathbb{N}$. Suppose that $x, y$ are vertices of $\Gamma_{R}(M)$ such that $d(x, y) \neq 1$. If $a x=0=a y$ we have $d(x, y) \leq 2$. Let $a x=0$ and $a y \neq 0$, so $a^{n-1} \in A n n(x) \cap A n n(y)$, hence $x-a y-y$ is a path of length 2 and $d(x, y) \leq 2$. If $a x \neq 0$ and $0=a y$, then $x-a x-y$ is a path of length 2 and $d(x, y) \leq 2$. Also if $a x \neq 0$ and $a y \neq 0$, then $x-a x-a y-y$ is a path of length 3 and $d(x, y) \leq 3$. Therefore $\operatorname{diam}\left(\Gamma_{R}(M)\right) \leq 3$.

The following example shows that $\Gamma_{R}(R)$ is complete in Theorem 3.1 (1) is crucial. 
Example 3.2. Let $R=\mathbb{Z}_{6}$ and $M=\mathbb{Z}_{6}$. Clearly $V\left(\Gamma_{R}(M)\right)=\{\overline{2}, \overline{3}, \overline{4}\}$ and vertex $\overline{3}$ is not adjacent to other vertices. This shows that $\Gamma_{R}(M)$ is not connected graph.

In the following example, it is shown that the condition $\operatorname{Nil}(R) \neq 0$ in Theorem 3.1 (3) cannot be omitted.

Example 3.3. Let $R=\mathbb{Z}_{6}$ and $M=\mathbb{Z}_{6} \oplus \mathbb{Z}_{3}$. Clearly

$$
V\left(\Gamma_{R}(M)\right)=\{(0, \overline{1}),(0, \overline{2}),(\overline{2}, \overline{0}),(\overline{2}, \overline{1})(\overline{3}, \overline{0}),(\overline{4}, \overline{0}),(\overline{4}, \overline{1}),(\overline{4}, \overline{2}),(\overline{5}, \overline{0})\} .
$$

It is easy to see that $(\overline{3}, \overline{0})$ is an isolated vertex and so $\Gamma_{R}(M)$ is not connected.

Corollary 3.4. If $R=\mathbb{Z}_{p^{n}}$, where $p$ is a prime number.

$$
Z(R)^{*}=\left\{\bar{p}, \overline{2 p}, \ldots, \overline{(p-1) p}, \overline{p^{2}}, \ldots, \overline{(p-1) p^{2}}, \ldots, \overline{p^{n-1}}, \ldots, \overline{(p-1) p^{n-1}}\right\} .
$$

Then $p^{n-1} \in \operatorname{Ann}(x) \cap \operatorname{Ann}(y)$, for every $x, y \in Z(R)^{*}$ and so $\Gamma_{R}(R)$ is a complete graph. Hence $\Gamma_{R}(M)$ is connected with diam $\left(\Gamma_{R}(M)\right) \leq 3$, for every $R$-module $M$.

Theorem 3.5. Let $M$ be a multiplication $R$-module and $N i l((R) \neq 0$. Then $\Gamma_{R}(M)$ is connected with $\operatorname{diam}\left(\Gamma_{R}(M)\right) \leq 2$.

Proof. Let $0 \neq a \in \operatorname{Nil}(R)$, so $a^{n}=0$ and $a^{n-1} \neq 0$ for some $n \in \mathbb{N}$. Suppose that $x, y$ are vertices of $\Gamma_{R}(M)$ such that $d(x, y) \neq 1$. If $[x: M] y \neq 0$, then there is $0 \neq \alpha \in[x: M]$ such that $x-\alpha y-y$ is a path of length 2 and so $d(x, y) \leq 2$. If $[y: M] x \neq 0$, then similar to the above argument, we have $d(x, y) \leq 2$. If $a x=a y=0$, then we have $d(x, y) \leq 2$. Let $a x=0$ and $a y \neq 0$, so $a^{n-1} \in A n n(x) \cap A n n(y)$, hence $x-a y-y$ is a path of length 2. Therefore $\operatorname{diam}\left(\Gamma_{R}(M)\right) \leq 2$.

Theorem 3.6. Let $M$ be a multiplication $R$-module with $T(M) \neq M$. Then the following hold:

(1) $\Gamma_{R}(M)$ is a complete graph if and only if $\Gamma_{R}(R)$ is a complete graph.

(2) If $R$ be a Bézout ring, then $\operatorname{diam}\left(\Gamma_{R}(R)\right)=\operatorname{diam}\left(\Gamma_{R}(M)\right)$.

Proof. (1) Let $\Gamma_{R}(M)$ be a complete graph and $A n n(m)=0$ for some $m \in$ $M$. Suppose that $\alpha, \beta$ are two vertices of $\Gamma_{R}(R)$. One can easily check that $\alpha m, \beta m \in T(M)^{*}$. Therefore $\operatorname{Ann}(\alpha m) \cap \operatorname{Ann}(\beta m) \neq 0$, so $r \alpha m=$ $r \beta m=0$ for some $0 \neq r \in R$. Hence $r \alpha=r \beta=0$ and $d(\alpha, \beta)=1$. Consequently $\Gamma_{R}(R)$ is a complete graph.

Now, let $\Gamma_{R}(R)$ be a complete graph, and $x, y \in T(M)^{*}$. So $\operatorname{Ann}(x) \neq 0$ and $\operatorname{Ann}(y) \neq 0$. Thus there are two non-zero elements $r, s \in R$ such 
that $r x=0=s y$. Hence $r[x: M]=0=s[y: M]$. So for all $\alpha \in[x: M]$ and $\beta \in[y: M]$ we have $r \alpha=0=s \beta$ and $\alpha, \beta$ are the vertices of $\Gamma_{R}(R)$. Therefore $0 \neq t \in \operatorname{Ann}(\alpha) \cap \operatorname{Ann}(\beta) \neq 0$. Let $x=\sum_{i=1}^{n} \alpha_{i} m_{i}$ and $y=\sum_{j=1}^{m} \beta_{j} m_{j}$, where $0 \neq \alpha_{i} \in[x: M], 0 \neq \beta_{j} \in[y: M]$. Hence $t \in A n n(x) \cap \operatorname{Ann}(y)$ and $d(x, y)=1$. Consequently $\Gamma_{R}(M)$ is a complete graph.

(2) Let $R$ be a Bézout ring and $M$ be a multiplication $R$-module. By $(1), \operatorname{diam}\left(\Gamma_{R}(M)\right)=1$ if and only if $\operatorname{diam}\left(\Gamma_{R}(R)\right)=1$. Suppose that $\operatorname{diam}\left(\Gamma_{R}(R)\right)=2$ and $x, y \in T(M)^{*}$ such that $d(x, y) \neq 1$. Let $x=\sum_{i=1}^{n} \alpha_{i} m_{i}$ and $y=\sum_{j=1}^{m} \beta_{j} m_{j}$, where $0 \neq \alpha_{i} \in[x: M], 0 \neq \beta_{j} \in$ $[y: M]$. Since $R$ is a Bézout ring, $\sum_{i=1}^{n} R \alpha_{i}=R \alpha$ and $\sum_{j=1}^{m} R \beta_{j}=R \beta$, for some $\alpha, \beta \in R$. Hence there exist $m, m_{0} \in M$ such that $x=\alpha m$, $y=\beta m_{0}$. Thus $\alpha, \beta \in Z(R)^{*}$. If $d(\alpha, \beta)=1$, then $d(x, y)=1$, and so we have a contradiction. Thus $d(\alpha, \beta)=2$, so there exists $\gamma \in Z(R)^{*}$ such that $\alpha-\gamma-\beta$ is a path of length 2 and there are non-zero elements $r, s \in R$ such that

$$
r \in \operatorname{Ann}(\alpha) \cap \operatorname{Ann}(\gamma), s \in A n n(\gamma) \cap \operatorname{Ann}(\beta)
$$

Since $M \neq T(M)$, then there is $n \in M$ such that $\gamma n \in T(M)^{*}$. Therefore

$$
r \in \operatorname{Ann}(x) \cap \operatorname{Ann}(\gamma n), s \in \operatorname{Ann}(\gamma n) \cap \operatorname{Ann}(y)
$$

and $\alpha m=x-\gamma n-y=\beta m$. is a path of length 2. So $d(x, y)=2$ and $\operatorname{diam}\left(\Gamma_{R}(M)\right)=2$.

Suppose that $\operatorname{diam}\left(\Gamma_{R}(M)\right)=2$ and $\alpha, \beta \in Z(R)^{*}$ such that $d(\alpha, \beta) \neq 1$. Since $M \neq T(M)$, there is $n \in M$ such that $\alpha n \neq 0$ and $\beta n \neq 0$. Hence $\beta n \neq \alpha n \in T(M)^{*}$. One can easily check that $d(\alpha n, \beta n) \neq 1$. So $d(\alpha n, \beta n)=2$, and there is $z=\gamma m \in T(M)^{*}$ such that $\alpha n-\gamma m-\beta n$, is a path of length 2. Thus $r \alpha n=0=r z$ for some $0 \neq r \in R$, so $r \gamma \in r[z: M]=0$, hence $\alpha-\gamma-\beta$ is a path of length 2 and $d(\alpha, \beta)=2$. Therefore $\operatorname{diam}\left(\Gamma_{R}(R)\right)=2$.

Now, by similar to above argument $\operatorname{diam}\left(\Gamma_{R}(R)\right)=n$ if and only if $\operatorname{diam}\left(\Gamma_{R}(M)\right)=n$. Consequently $\operatorname{diam}\left(\Gamma_{R}(M)\right)=\operatorname{diam}\left(\Gamma_{R}(R)\right)$.

\section{Girth of torsion graph}

In this section we study the girth of torsion graph.

Theorem 4.1. Let $M$ be an $R$-module. If $\Gamma_{R}(M)$ contains a cycle, then $\operatorname{gr}\left(\Gamma_{R}(M)\right)=3$. 
Proof. Let $x-y-z-w-x$ be the shortest cycle of $T(M)$, so there are non-zero elements $r, s$ such that $r \in \operatorname{Ann}(x) \cap \operatorname{Ann}(y)$ and $s \in \operatorname{Ann}(y) \cap \operatorname{Ann}(z)$. If $x+y=0$, then $s \in \operatorname{Ann}(x) \cap \operatorname{Ann}(z)$ and so $x-y-z-x$ is a cycle, which is a contradiction. Hence suppose that $x+y \neq 0$, we know that $r \in A n n(x) \cap$ $\operatorname{Ann}(x+y)$ and $s \in A n n(x+y) \cap \operatorname{Ann}(y)$. Thus $\Gamma_{R}(M)$ contains a cycle $x-x+y-y-x$ which is a contradiction. Consequently, $\operatorname{gr}\left(\Gamma_{R}(M)\right)=3$.

As a result of Theorem4.1, we could say that the torsion graph of $R$-module $M$ can not be an n-gon for $n \geq 4$.

Corollary 4.2. Let $M$ be an $R$-module. If $\Gamma_{R}(M)$ is a connected graph with $\left|\Gamma_{R}(M)\right|>2$, then $\Gamma_{R}(M)$ contains a cycle and $\operatorname{gr}\left(\Gamma_{R}(M)\right)=3$

Proof. Let $\Gamma_{R}(M)$ is a connected graph with $\left|\Gamma_{R}(M)\right|>2$. Suppose that $x-y-z$ be the path in $\Gamma_{R}(M)$. By the same argument as in the proof of Theorem 4.1, and if $x+y=0$, then $\Gamma_{R}(M) x-y-z-x$ is a cycle, and if $x+y \neq 0$, we have $\Gamma_{R}(M)$ contains a cycle $x-x+y-y-x$. Consequently, $\Gamma_{R}(M)$ contains a cycle and so $\operatorname{gr}\left(\Gamma_{R}(M)\right)=3$.

Theorem 4.3. Let $M$ be a faithful multiplication $R$-module. Then $\operatorname{gr}\left(\Gamma_{R}(M)\right)=\operatorname{gr}\left(\Gamma_{R}(R)\right)$.

Proof. Let $M$ be a faithful multiplication $R$-module. We show that $\Gamma_{R}(M)$ contains a cycle if and only if $\Gamma_{R}(M)$ contains a cycle. Let $\Gamma_{R}(M)$ contains a cycle, by Theorem $4.1 \operatorname{gr}\left(\Gamma_{R}(M)\right)=3$. So there are $x, y, z \in T(M)^{*}$ such that $x-y-z-x$ is a cycle. Hence $r \in \operatorname{Ann}(x) \cap \operatorname{Ann}(y), s \in \operatorname{Ann}(y) \cap \operatorname{Ann}(z)$ and $t \in \operatorname{Ann}(z) \cap \operatorname{Ann}(x)$ for some $r, s, t \in R \backslash\{0\}$. Therefore for all $\alpha \in$ $[x: M], \beta \in[y: M]$ and $\gamma \in[z: M]$ we have $r \in \operatorname{Ann}(\alpha) \cap \operatorname{Ann}(\beta)$, $s \in \operatorname{Ann}(\beta) \cap \operatorname{Ann}(\gamma)$ and $t \in \operatorname{Ann}(\gamma) \cap \operatorname{Ann}(\alpha)$. Thus $\alpha-\beta-\gamma-\alpha$ is a cycle in $\Gamma_{R}(R)$. So $\operatorname{gr}\left(\Gamma_{R}(R)\right)=3$. Conversely, suppose that $\alpha-\beta-\gamma-\alpha$ is a cycle in $\Gamma_{R}(R)$. Since $M$ is faithful, there are non-zero elements $m_{1}, m_{2}, m_{3} \in M$ such that $\alpha m_{1}, \beta m_{2}, \gamma m_{3} \in T(M)^{*}$. Therefore $\alpha m_{1}-\beta m_{2}-\gamma m_{3}-\alpha m_{1}$ is a cycle in $\Gamma_{R}(M)$ and so $\operatorname{gr}\left(\Gamma_{R}(M)\right)=3$.

\section{ACKNOWLEDGMENTS}

The first and fourth authors are indebted to Department of Mathematics, Islamic Azad University, Qazvin Branch, Qazvin, Iran for support. The research of the first and fourth authors were in part supported by a grant from Islamic Azad University, Qazvin Branch. 


\section{References}

[1] D. F. Anderson, A. Badawi, The total graph of a commutative ring, $J$. Algebra 320 (2008) 27062719.

[2] D. F. Anderson, A. Badawi, On the zero-divisor graph of a ring, Comm. Algebra 36 (2008) 30733092.

[3] D.F. Anderson, A. Frazier, A. Lauve, and P.S. Livingston, The zerodivisor graph of a commutative ring, II. in, Lecture Notes in Pure and Appl. Math., Marcel Dekker, New York,220 (2001) 61-72.

[4] D.F. Anderson, R. Levyb, J. Shapirob, Zero-divisor graphs, von Neumann regular rings, and Boolean algebras. J. Pure Appl. Algebra 180 (2003) $221-241$

[5] D.F. Anderson and P.S. Livingston, The zero-divisor graph of a commutative ring, J. Algebra 217 (1999) 434-447.

[6] D.F. Anderson, Sh. Ghalandarzadeh, S. Shirinkam, P. Malakooti Rad, On the diameter of the graph $\Gamma_{A n n(M)}(R)$, Filomat. 26 (2012) 623-629.

[7] F.W. Anderson, K.R. Fuller, Rings and Categories of Modules, SpringerVerlag 1992.

[8] M.F. Atiyah and I.G. MacDonald, Introduction to Commutative Algebra. Addison-Wesley, Reading, MA, 1969.

[9] A. Badawi, D. F. Anderson, Divisibility conditions in commutative rings with zero divisors. Comm. Algebra 38 (2002) 40314047.

[10] I. Beck, Coloring of commutative rings, J. Algebra 116 (1988) 208-226.

[11] A. Cannon, K. Neuerburg, S.P. Redmond, Zero-divisor graphs of nearrings and semigroups. in, Kiechle, H. Kreuzer,A., Thomsen, M.J. (Eds.), Nearrings and Nearfields, Springer, Dordrecht, The Netherlands, (2005) 189-200.

[12] R. Diestel, Graph Theory. Springer-Verlag, New York, (1997).

[13] Z.A. El-Bast, P.F. Smith, Multiplication modules. comm. Algebra 16 (1988) $755-779$.

[14] Sh. Ghalandarzadeh, S. Shirinkam, P. Malakooti Rad, Annihilator IdealBased Zero-Divisor Graphs Over Multiplication Modules, Communications in Algebra. 41 (2013) 1134-1148. 
[15] D.C. Lu, T.S. Wu, On bipartite zero-divisor graphs, Discrete Math. 309 (2009) 755-762.

[16] P. Malakooti Rad, Sh. Ghalandarzadeh, S. Shirinkam, On The Torsion Graph and Von Numann Regular Rings, Filomat. 26 (2012) 47-53.

[17] S.P. Redmond, The zero-divisor graph of a non-commutative ring, Internat. J. Commutative Rings 1 (2002) 203-211.

Parastoo Malakooti Rad

Department of Mathematics,

Islamic Azad University,

Qazvin Branch, Qazvin, Iran.

Email: pmalakoti@gmail.com

Siamak Yassemi

School of Mathematics,

Statistics and Computer Science,

College of Science, University of Tehran, Tehran, Iran,

and School of Mathematics,

Institute for Research in Fundamental Sciences (IPM), P.O. Box 19395-5746,

Tehran, Iran

Email: yassemi@ipm.ir

Shaban Ghalandarzadeh

Department of Mathematics,

Faculty of Science,

K. N. Toosi University of Technology, Tehran, Iran.

Email: ghalandarzadeh@kntu.ac.ir

Parvin Safari

Department of Mathematics,

Islamic Azad University,

Qazvin Branch, Qazvin, Iran.

Email: ms.p.sapari@gmail.com 